\title{
Statistical Study of Solar Eruptive Events Observed with Nobeyama Radioheliograph at $17 \mathrm{GHz}$
}

\author{
K. Hori \\ Mullard Space Science Laboratory, University College London, \\ Holmbury St. Mary, Dorking, Surrey, RH5 6NT, UK
}

\begin{abstract}
We report statistical features of 49 eruptive prominences observed near solar maximum (1999-2000) with Nobeyama Radiohelograph. Here we focus on the location and related phenomena of the prominence activity. By using white light synoptic maps from LASCO C2 coronagraph, we found that most of the events are associated with some heating process taking place around the prominence site, and occur along pre-existing streamers associated with CMEs as well as small mass ejections. These features suggest that streamers connect the activity on the solar surface toward the outer corona.
\end{abstract}

\section{Introduction}

Since June 1992, Nobeyama Radioheliograph (NoRH; Nakajima et al. 1994) has observed many eruptive prominences visible in microwaves $(17 \mathrm{GHz})$. Extending $\mathrm{H} \alpha$ observations, NoRH can monitor prominence activity continuously over a wide range of velocities and temperatures $\left(\mathrm{a}\right.$ few $\left.\times 10^{3-4} \mathrm{~K}\right)$. So far we have found that i) most prominence eruptions are not associated with GOES soft $\mathrm{X}$-ray flares and ii) the eruptions tend to occur at the latitude from which dense streamers emanated beforehand (Hori 2000). What kind of phenomena proceed prominence activity? What is the role of streamers in the activation of a prominece? Here we summarize our survey of NoRH eruptive prominences that can provide characteristics of mass ejections from the Sun.

\section{Observations and Results}

At $17 \mathrm{GHz}$, low temperature plasmas such as filaments (prominences) can be seen in absorption on the disk whilst appearing in emission above the limb. We surveyed eruptive prominences located at, or erupting above, the limb from NoRH daily observations during the period from February 1999 to May 2000. Next, we selected 49 events that were also observed with both SOHO LASCO C2 coronagraph (Brueckner it et al. 1995) and Yohkoh SXT (Tsuneta et al. 1991). In order to see the extent of the coronal activity, we made white light synoptic maps from LASCO C2 images (level 0.5) by taking a slice of each C2 image at a height of 2.5 Rs. C2 observes the outer corona within 2-6 Rs from the Sun center at about 10-60 minute cadence. By Spline-fitting the observed slices, we made the synoptic maps with slices at 40 second cadence. 
Figure 1 shows the March 16-17, 1999 event. At the NW limb, the prominence gradually erupted twice toward different directions (see arrows). A CME with a circular pattern was observed in association with eruption $B$ (a). By overlaying the latitude of the prominence tips on a C2 synoptic map, it can be seen that there is an one-to-one correspondence between the prominence motion and the CME motion above. Eruption $A$ occurred along the southern edge of the pre-existing streamers (a), which later became the core of the CME (c). Eruption $B$ traces the circular motion of the CME, which disappeared at the latitude of the northern edge of the streamers $(b, c)$. Both prominence and CME motions are banded by a bundle of (disrupting) streamers. During the event, many $\mathrm{C} 1$-class flares occurred at the AR near the prominence but the relation between the two phenomena is not clear.

The initial activity for most of the eruptive prominences can be found in the lower corona or in the chromosphere around the prominence site. However, we found 7 events in which prominences were activated through streamers disturbed by a preceding CME. Most of the prominence eruptions are associated with some energy release, e.g., GOES flares $(16 \%$, plus $20 \%$ if neighborhood flares are included), an increase of the brightness temperature at $17 \mathrm{GHz}$ in the prominence $(26 \%)$, and post-eruption soft X-ray features like an arcade or loops $(29 \%)$. CMEs were observed in $92 \%$ of the events, while a weak plasma motion was observed in streamers for the rest of the events. Both prominences and CMEs moved along pre-existing streamers or in sparse regions sandwiched by streamers. The average speed of the prominence eruptions was rather low; $80 \mathrm{~km} \mathrm{sec}^{-1}$.

\section{Conclusion}

While prominence activity is usually triggered by some energy release process taking place near the solar surface, that can be triggered by large coronal disturbances far from the prominence site. By monitoring the activity of microwave prominences, we can suppose global coronal fields emanating from the solar surface. The close relation between streamers and CMEs (Hundhausen 1993; Subramanian et al. 1999) is supported by our high cadence white light synoptic maps. On the basis of the current study, the role of streamers as a corridor of mass ejections from the Sun will be discussed in the next paper.

The author greatly benefits from the open data policy of the SoHO, Yohkoh, and Nobeyama Radioheliograph team. This work was supported by PPARC.

\section{References}

Brueckner, G. E., et al., 1995, Solar Phys., 162, 357.

Hori, K. 2000, ApJ, 543 (in press)

Hundhausen, A. J. 1993, J. Geophys. Res., 98, 13177.

Nakajima, H., et al., 1994, Proceedings of the IEEE, 82, 705.

Subramanian, P., et al., 1999, J. Geophys. Res., 104, 22321.

Tsuneta, S., et al., 1991, Solar Phys., 136, 37. 

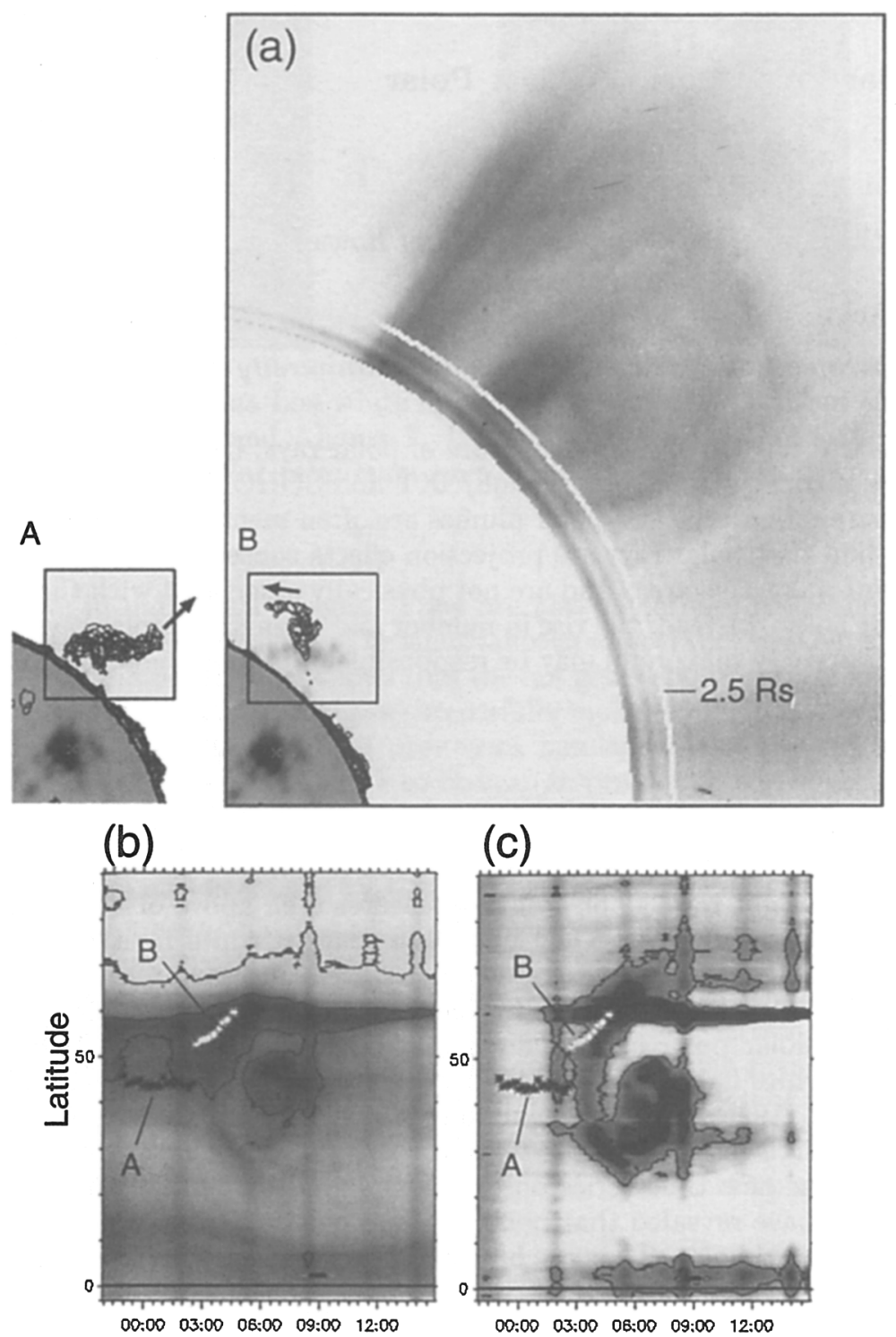

Figure 1. Prominence eruption and CME on March 16-17, 1999. (a) C2 image at 06:54 UT on March 17 (outer), and NoRH $17 \mathrm{GHz}$ images with contours at $\mathrm{T}_{b}=[0.5,1.0] \times 10^{4} \mathrm{~K}$ taken 23:01-02:41 (A) and 02:51-05:21 (B), respectively. (b) West limb (0-90 N) C2 synoptic map at $2.5 \mathrm{Rs}$, with latitude of the prominence tips overlaid on it (asterisks $A$ and B). (c) The initial slice in (b) is subtracted from (b). 